\title{
Nakajo-Nishimura syndrome
}

INSERM

\section{Source}

INSERM. (1999). Orphanet: an online rare disease and orphan drug data base. NakajoNishimura syndrome. ORPHA:2615

Nakajo-Nishimura syndrome (NNS) is a rare autoinflammatory disorder belonging to the proteasome disability syndrome (see this term) group, and characterized by pernio-like lesions appearing in infancy followed by recurrent fever, nodular skin eruption, partial lipodystrophy (mainly in upper extremities and face) and joint contractures. 